\title{
THE USE OF CALCIUM HYDROXIDE AS AN INTRACANAL MEDICAMENT IN THE TREATMENT OF LARGE PERIAPICAL LESIONS. A REVIEW
}

\author{
TIMEA DAKO ${ }^{1}$, MIHAI POP ${ }^{2}$, JULIA FULOP ${ }^{3}$, JANOS KANTOR ${ }^{4}$, MONICA MONEA $^{5}$ \\ ${ }^{1,3,4}$ County Emergency Clinical Hospital of Târgu-Mureș, \\ 2,5 “George Emil Palade” University of Medicine, Pharmacy, Science and Technology of Târgu-Mureș
}

\begin{abstract}
Keywords: $\quad$ calcium Abstract: Calcium hydroxide is a slow-acting antiseptic substance used in several forms for the last hydroxide, endodontics, century in various fields of dentistry. Its applications in endodontics are by far the most meaningful large periapical lesion, including the treatment of root resorptions and perforations, inducing the apexification process, and intracanal medicament most importantly as intracanal medicaments representing a crucial step in the nonsurgical management of large periapical lesions. The aim of this article is to conduct a review of the properties, antimicrobial effect, combination with adjuvant substances, biocompatibility, cytotoxicity, and adverse effects of calcium hydroxide-based dressings as an additional manoeuvre in the conservative treatment of chronic apical periodontitis and to draw attention on the importance of this extra step.
\end{abstract}

\section{INTRODUCTION}

Calcium hydroxide is a widely used substance in dentistry since its first introduction by Hermann in 1920 as a pulp-capping agent. Since then, its usage range has enlarged, being adopted as an inter-visit dressing for root canals, due to its significant disinfecting properties which in the long run are able to ensure periapical healing.(1) The main purpose of an endodontic treatment, especially in teeth with associated periapical disease, is to completely eradicate intraradicular bacteria and infected pulp residue as well as a proper apical seal of these root canals. These disinfecting outlines were thought to be reached by the imperative step of using intracanal medicaments during or in between visits, even though modern endodontics affirms that thorough cleaning and shaping are of a greater importance than intracanal dressings in the successful outcome of endodontic treatments. However, intracanal medicaments are still used nowadays especially when the endodontic treatment requires more than one appointment for the shaping of the root canals and most likely in the presence of a large periapical granuloma or cyst.(2) It is a well-known fact that teeth with associated periapical disease are correlated with higher failure percentage after a root canal treatment, thus the necessity of applying an intracanal dressing for multiple visits to enhance disinfection of the endodontic anatomy and the periapical tissue. Numerous other measures have been introduced over time to decrease the level of microorganisms from the endodontic system including various irrigation regimes and different mechanical instrumentation procedures, but as a multitude of studies have shown, these two alone cannot provide enough disinfection of the root canals. With the aid of root canal dressing materials, the success rate of endodontic treatments can be considerably improved, and surgical treatment could be avoided.(3) Among various substances used for this purpose, calcium hydroxide is the most approved by specialists in the endodontic field. From a chemical point of view, calcium hydroxide $\left(\mathrm{Ca}(\mathrm{OH})_{2}\right)$ is a white odourless powder, categorized as a strong base, having a molecular weight of 7.08.

It is supplied in mainly three forms:

1. as a liquid containing calcium hydroxide suspended in a solvent and used like a varnish

2. as a paste consisting of calcium hydroxide suspended in methylcellulose

3. as base and catalyst forming a hard compound when combined

Numerous studies have documented the beneficial effect of using $\mathrm{Ca}(\mathrm{OH})_{2}$ as an inter-visit dressing due to its disinfecting properties of the root canals in only 7 days' time. This antimicrobial property is based upon its strongly alkaline $\mathrm{pH}(12.5-12.8)$ which is maintained for several weeks by its low solubility in water and periapical fluids. Its mechanism of action is related to the dissociation of calcium and hydroxyl ions on contact with a watery solution. Tronstadt et al pointed out that once introduced into the root canal system, calcium hydroxide liberates hydroxyl ions through the dentinal tubules, fixing a high $\mathrm{pH}$ of 9 on the root surface. This alkaline environment produced by elevating the $\mathrm{pH}$ level due to the slow-dissolving action of calcium hydroxide is unfavourable for most pathogens which are unable to survive in highly alkaline environments. Therefore, the high $\mathrm{pH}$ level provided by calcium hydroxide has bactericide assets and inhibits any osteoclastic activity. Furthermore, the diffusion of the hydroxyl ions can be improved by eliminating the smear-layer from the root canal walls by irrigating with a $17 \%$ EDTA solution. (4)

\section{Properties of calcium hydroxide}

Calcium hydroxide is widely used in endodontics, being suitable for the non-surgical treatment of large periapical lesions due to its advantages such as:

- Highly alkaline $\mathrm{pH}$ levels which are initially bactericidal $(\mathrm{pH}=12)$ then bacteriostatic ( $\mathrm{pH}$ decreases to $9-10$ after 4 weeks) and which also stimulate fibroblasts

\footnotetext{
${ }^{2}$ Corresponding author: Mihai Pop, Str. Ghe. Marinescu, Nr. 38, Târgu-Mureş, România, E-mail: mihaipop_99@yahoo.com, Phone: +40723 000227
} Article received on 08.04.2020 and accepted for publication on 02.06 .2020 


\section{CLINICAL ASPECTS}

- Impressive broad-spectrum antimicrobial activity identified ex vivo, killing $99.9 \%$ of bacteria in only 1 to 6 minutes by damaging their membrane and DNA and denaturing their proteins

- Anti-inflammatory effect reducing periapical exudate

- Inhibits internal resorption and promotes healing and repair of the periodontal lesions and hard tissue formation

- Eliminates persistent discharge from periapical lesions ensuring a dry environment for the furthered step of final root canal filling (3)

\section{Antimicrobial effect of calcium hydroxide}

The endodontic microbiota of large periapical lesions most frequently consists of the following microorganisms: Peptococcus, Peptostreptococcus, Prevotella, Porphyromonas, Eubacterium, Streptococcus, Lactobacillus and Fusobacterium. Several facultative bacteria have also been associated with chronic periapical disease such as Enterococcus faecalis. Moreover, Candida albicans was also discovered to be part of the pathogens that cause and maintain apical periodontitis. Sjogren et al studied the in vivo effect of calcium hydroxide as a short-term intracanal dressing achieving successful results as all bacteria from all root canals was eradicated in only 7 days. Several studies have investigated the in vivo and in vitro effect of calcium hydroxide on different pathogens linked to periapical radiolucencies, some of which are presented below (table no. 1).(2)

Table no. 1. Effect of calcium hydroxide on different pathogens

\begin{tabular}{|c|c|c|c|}
\hline Year & Researcher & Pathogen & Result \\
\hline 1991 & Stuart et al & Actinomyces Viscosus & - \\
\hline 2002 & Gomes et al & Prevotella intermedia & + \\
\hline 2002 & Gomes et al & Porphyromonas e. & ++ \\
\hline 2010 & Delgado et al & E. faecalis & $+/-$ \\
\hline 2011 & Gangwar et al & S. aureus, Candida a. & ++ \\
\hline 2011 & Gangwar et al & E. faecalis & $+/-$ \\
\hline 2012 & Dianat et al & Lactobacillus r. & + \\
\hline 2019 & Rao S et al & E. faecalis, S. mutans & + \\
\hline \multicolumn{4}{|c|}{++ major effect + good effect } \\
\hline \multicolumn{4}{|c|}{$+/$ limited effect - no effect } \\
\hline
\end{tabular}

Although, some in vitro studies showed limited or zero effect of calcium hydroxide-based intracanal dressings on some microorganisms such as E. faecalis or Candida albicans, several in vivo papers (Weiger et al, Caliskan et al) reported high success rates $(85-90 \%)$ in the healing of teeth with associated periapical disease which were followed up for several years. This fact is of a greater importance than the low results obtained by in vitro studies as they cannot be compared to the individuality of each patient's immune response cooperating with the beneficial effect of calcium hydroxide. $(3,4)$ Furthermore, Fernandes et al suggested that using calcium hydroxide paste beyond the apex had great success in cases with large periapical lesions. He also pointed out that the size of a periapical lesion is not the decisive component in making the decision to perform surgical eviction or to adopt the conservative management. Even large granulomas or cysts can wholly heal following non-surgical endodontic treatment. $(5,6)$

\section{Combination with adjuvant substances}

Various works have studied the influence of other substances in combination with calcium hydroxide in the healing course of large periapical lesions, as several authors suggested the inactivation of its antibacterial activity during its linger in the endodontic system. Therefore, diverse vehicles were incorporated into $\mathrm{Ca}(\mathrm{OH})_{2}$ pastes in attempt to improve its antimicrobial effect against different pathogens responsible for maintaining periapical infection. Among these additional substances, iodoform has proved itself to be highly effective in the conservative treatment approach of large periapical lesions. Iodoform's action occurs by liberating iodine which has high antibacterial reactivity based on oxidizing enzymes and precipitating proteins.(7) Its important infection control and considerable disinfecting properties are the reason why iodoform is such a popular choice when it comes to incorporating them into calcium hydroxide pastes. According to Estrela et al, $\mathrm{Ca}(\mathrm{OH})_{2}$ associated with iodoform and saline showed antimicrobial effectiveness on S. aureus, E. faecalis, P. aeruginosa, B. subtilis and C. albicans.(8) Other surveys (Khasawnah et al) also revealed bone regenerative capacity of calcium hydroxide-iodoform intracanal dressing pastes, showing complete healing of large periapical lesions in approximately 75 days.(9)

Silver nanoparticles have several beneficial medical applications as a result of their anti-inflammatory and antibacterial properties. According to current knowledge, these are based upon the release of silver ions which lead to structural changes of the bacterial wall due to the high affinity of silver ion's binding protein to those from the bacterial membrane. Recent studies revealed that calcium hydroxide combined with silver nanoparticles as an intracanal medicament, has strong bactericidal potential against $\mathrm{E}$. faecalis, the main etiological pathogen correlated with periapical infection.(10)

Fuzz et al and Knappwost et al also reported that the antibacterial properties of calcium hydroxide and copper combination were effective on E. faecalis by showing no viable bacteria in dentinal tubules after one week's application.(11) It should be kept in mind that the antibacterial efficacy of these metal components revolve around their contact surface, which is currently facilitated by reducing particle sizes from micro to nanoscale with the progress made by modern technology. Among other substances that boost the antibacterial properties of calcium hydroxide are zinc oxide and magnesium oxide as shown by Yousefshani et al.(12) While $\mathrm{Ca}(\mathrm{OH})_{2}$ alone is efficacious on all periapical infections, as far as large periapical lesions are concerned, all resources available should be exploited in the nonsurgical management of these situations, including associating calcium hydroxide with diverse adjuvant substances.

Time necessary for calcium hydroxide to induce healing

Several authors conducted studies on the time needed for calcium hydroxide-based dressings to completely eradicate pathogens associated with periapical lesions and promote bone healing. While Estrela et al claim a minimum of 60 days necessary for this process, Han et al declare, based on their in vitro research, that a 3-week period is required for the same effect. According to Sjogren et al, at most 7 days are necessary for the destruction of microorganisms from infected root canals of periapical lesions.(13)

Although periapical bone healing for smaller lesions can take up to 6 months, large periapical lesions require 9 to 12 months for complete bone regeneration around the apical region as the average healing rate is about $3 \mathrm{~mm}^{2} /$ month. The intracanal medicament for these extensive bone destroying lesions should be placed for at least 1 to 2 months and refreshed once every few weeks, according to Dixit et al (14) accompanied by followup examinations which are essential for at least 2 years (15) until radiographic evaluations demonstrate bone regeneration justified by increasing density, trabecular reconstruction and lamina dura forming.(16)

\section{Biocompatibility and cytotoxicity}

Materials used in endodontics come into direct contact with at least two types of tissues such as the dentinal walls of the root canals and the periapical tissue which in the presence of large periapical lesions, is histologically modified by the 


\section{CLINICAL ASPECTS}

endodontic microbiota and requires thorough disinfection. The principle of every material used for root canal therapy is not to be cytotoxic and to induce the periapical tissues to repair bone destruction. As Mori et al reported in their in vivo study, nonsignificant or mild inflammatory reaction was revealed by analysing histological sections of subcutaneous tissue that was in contact with calcium hydroxide-based pastes for 30 days.(17) Similar results were outlined by Silva et al and Andolfatto et al, which prove that calcium hydroxide is a biocompatible substance, very important aspect in the management of teeth associated with large periapical lesions as the dressing material should not cause additional inflammatory stress upon the periapical region. $(18,19)$

\section{Short and long-term adverse effects}

Several studies from international literature have suggested that long-term and short-term contact of calcium hydroxide and root dentin can make changes in the strength of the last-mentioned and potentially cause root fracture. This statement is based upon the so-said disturbance of collagen and hydroxyapatite network of root dentin caused by calcium hydroxide, described by Sahebi et at.(18,20) This can be a real concern in the management on large periapical lesions which require multiple weeks of intracanal medicaments in order for the bone repairing process to be induced. Contrary to the conclusion of Sahebi's study, Kahler et al demonstrated no significant difference between month long exposed radicular dentin to calcium hydroxide and negative controls. Furthermore, the root fracture was established to be caused by thin and fragile roots rather than long-time exposure to $\mathrm{Ca}(\mathrm{OH})_{2}$-based dressings.(20) A concerning long-term side effect which can play a negative impact of the favourable outcome of large periapical lesions' treatment is the remaining calcium hydroxide from the root canal walls. This can restrict the penetration of root canal sealer into the dentinal tubules hindering a watertight seal of the root canal filling. Therefore, the complete removal of the calcium hydroxide-based dressings is advised for a positive endodontic outcome, especially in teeth associated with large cyst-like lesions. Several researchers investigated the removal of $\mathrm{Ca}(\mathrm{OH})_{2}$ using different techniques and products and came to the conclusion that the best irrigation protocol is with sodium hypochlorite followed by EDTA rinsing achieved by using the Endoactivator.(19)

\section{CONCLUSIONS}

$\mathrm{Ca}(\mathrm{OH})_{2}$ based intracanal medicaments are a crucial step in the non-surgical management of large periapical lesions. Along with sufficient mechanical cleaning of the endodontic system and copious irrigation using sodium hypochlorite, these three steps can assure a satisfactory outcome of extensive bone destroying lesions. Considering calcium hydroxide's broadspectrum antimicrobial properties, biocompatibility, low cytotoxicity and enhanced effect when combined with other anti-inflammatory substances, it should be the first option when considering treatment methods for large periapical lesions.

\section{REFERENCES}

1. Kim D, Kim E. Antimicrobial effect of calcium hydroxide as an intracanal medicament in root canal treatment: a literature review - Part I. In vitro studies, Rest Dent and Endo. 2014, Nov;39(4), 241-252.

2. Roman I, Bocskay S, Torcătoru A. Patologia si terpaia cariei complicate. Endodontie. University Press TârguMures; 2009. p. 255-8.

3. Caliskan MK, Sen BH. Endodontic treatment of teeth with apical periodontitis using calcium hydroxide: a long-term study. Endo \& Dent Traum. 1996;12(5):215-21.
4. Weiger R, Resendahl R, Lost C. Influence of calcium hydroxide intracanal dressings on the prognosis of teeth with endodontically induced periapical lesions, Int Endo J. 2000;33(3):219-226

5. Fernandes M, Ataide Id. Nonsurgical management of periapical lesions, J Cons Dent. 2010;13(4):240-5.

6. Caliskan M, Prognosis of large cyst-like periapical lesions following nonsurgical root canal treatment: A cinical review. Int Endo J. 2004;37(6):408-16.

7. Estrela C, Holland R. Calcium hydroxide: study based on scientific evidences. J Appl Oral Sci. 2003;11:269-82.

8. Estrela C Estrela CR, Hollanda AC, Decurcio DdeA, Pecora JD. Influence od iodoform on antimicrobial potential of calcium hydroxide. J Appl Oral Scienc. 2006;14(1):33-7.

9. Khasawah QA, Hassan F, Malhan D. Nonsurgical Clinical Management of Periapical Lesions Using Calcium Hydroxide-Iodorom-Silicone-oil Paste. Biomed Research International; 2018.

10. Alabdulmohsen ZA, Saad AY. Antibacterial effect of silver nanoparticles against Enterococcus faecalis. Saudi Endo Jo. 2017;7:29-35.

11. Fuss Z, Mizrahi A, Lin S, A laboratory study of the effect of calcium Hydroxide mixed with iodince or electrophoretically activated copper on bacteria viability in dentinal tubules. Int Endo J. 2012;35(6):522-6.

12. Yousefshahi H, Aminsobhani M, Shokri M, Shahbazi R. Antibacterial properties of calcium hydroxide in combination with silver, copper, zinc oxide and magnesium oxide. Euro J Trans Myol. 2018;28(3):7545.

13. Estrela C, Rodrigues dAEC, Pecora J. A study of the time necessary for calcium hydroxide to eliminate microorganisms in infected canals. J Appl Oral Scien; 2003.

14. Dixit A, Dixit A, Kumar P. Nonsurgical treatment of two periapical lesions with calcium hydroxide using two different vehicles. Case Reports in Dentistry. 2014;2014:901497. doi: 10.1155/2014/901497. Epub 2014 Jul 15.

15. Mori GG, Rodrigues Sda S, Shibayama ST, Pomini M, doAmaral CO. Biocompatibility of a calcium hydroxidepropolis experimental paste in rat subcutaneous tissue. Braz Dent J. 2014;25(2):104-8

16. Silva RA, Assed S, Nelson-Filho P, Silva LA, Consolaro A. Subcutaneous tissue response of isogenic mice to calcium hydroxide-based pastes with chlorhexidine. Braz Dent J. 2009;20(2):99-106.

17. Andolfatto C, da Silva GF, Cornelio AL. Biocompatibility of intracanal medications based on calcium hydroxide. ISRN Dentistry; 2012.

18. Kawamoto R, Kurakawa H, Takubo C, Shimamura Y, Yoshida T, Miyazaki M. Change in elastic modulus of bovine dentine with exposure to a calcium hydroxide paste. J Dent. 2008;36(11):959-64.

19. Kahler SL, Shetty S, Andreasen FM, Kahler B. The effect of long-term dressing with calcium hydroxide on the fracture susceptibility of teeth. J Endod. 2018;44(3):464-9.

20. Salgado RJ, Moura-Netto C, Yamazaki AK. Comparison of different irrigants on calcium hydroxide medication removal: microscopic cleanliness evaluation. Oral Surg Oral Med Oral Pathol Oral Radiol Endo. 2009;107(4):5084. 